\title{
Article \\ Molecular Epidemiology of Fosfomycin Resistant E. coli from a Pigeon Farm in China
}

\author{
Lu Han ${ }^{1,2,+}{ }^{+}$Xiao-Qing Lu ${ }^{1,2,+}{ }^{\text {, Xu-Wei Liu }}{ }^{1,2}$, Mei-Na Liao ${ }^{1,2}$, Ruan-Yang Sun ${ }^{1,2}$, Yao Xie ${ }^{1,2}$, Xiao-Ping Liao ${ }^{1,2,3}$, \\ Ya-Hong Liu ${ }^{1,2,3}$, Jian Sun ${ }^{1,2,3}$ and Rong-Min Zhang ${ }^{1,2,3, *}$ \\ 1 National Risk Assessment Laboratory for Antimicrobial Resistance of Animal Original Bacteria, \\ College of Veterinary Medicine, South China Agricultural University, Guangzhou 510642, China; \\ luhan@stu.scau.edu.cn (L.H.); lxq@stu.scau.edu.cn (X.-Q.L.); xwliu11@stu.scau.edu.cn (X.-W.L.); \\ lmn@stu.scau.edu.cn (M.-N.L.); sunruanyang@163.com (R.-Y.S.); 2017zrm@stu.scau.edu.cn (Y.X.); \\ xpliao@scau.edu.cn (X.-P.L.); lyh@scau.edu.cn (Y.-H.L.); jiansun@scau.edu.cn (J.S.) \\ 2 Guangdong Provincial Key Laboratory of Veterinary Pharmaceutics Development and Safety Evaluation, \\ College of Veterinary Medicine, South China Agricultural University, Guangzhou 510642, China \\ 3 Guangdong Laboratory for Lingnan Modern Agriculture, College of Veterinary Medicine, South China \\ Agricultural University, Guangzhou 510642, China \\ * Correspondence: zrm@scau.edu.cn \\ + These authors contributed equally to this work.
}

Citation: Han, L.; Lu, X.-Q.; Liu, X.-W.; Liao, M.-N.; Sun, R.-Y.; Xie, Y.; Liao, X.-P.; Liu, Y.-H.; Sun, J.; Zhang, R.-M. Molecular Epidemiology of Fosfomycin Resistant E. coli from a Pigeon Farm in China. Antibiotics 2021, 10, 777. https://doi.org/ 10.3390/antibiotics10070777

Academic Editor: Qinglin Chen

Received: 22 May 2021

Accepted: 23 June 2021

Published: 25 June 2021

Publisher's Note: MDPI stays neutral with regard to jurisdictional claims in published maps and institutional affiliations.

Copyright: (C) 2021 by the authors. Licensee MDPI, Basel, Switzerland. This article is an open access article distributed under the terms and conditions of the Creative Commons Attribution (CC BY) license (https:// creativecommons.org/licenses/by/ $4.0 /)$.

\begin{abstract}
We determined the prevalence and molecular characteristics of fosfomycin-resistant Escherichia coli from a domestic pigeon farm. A total of 79 samples collected from pigeons and their surrounding environments were screened for the presence of fosfomycin resistant isolates and these included $49 \mathrm{E}$. coli isolates that displayed high-level resistance (MIC $\geq 256 \mathrm{mg} \mathrm{L}^{-1}$ ) and carried the fos $A 3$ gene on plasmids with sizes ranging from 80 to $370 \mathrm{~kb}$. MLST analysis of these fosA3-positive E. coli isolates indicated the presence of nine sequence types (ST6856, ST8804, ST457, ST746, ST533, ST165, ST2614, ST362 and ST8805) of which ST6856 was the most prevalent (24.5\%, 12/49). PFGE combined with genomic context comparative analyses indicated that the fos $A 3$ gene was spread by horizontal transfer as well as via clonal transmission between $E$. coli in the pigeon farm, and IS26 played an important role in fos $A 3$ transmission. The high prevalence of fos $A 3$ in the pigeon farm and the high similarity of the fos $A 3$ genomic environment between $E$. coli isolates from humans and pigeons indicated that the pigeon farm served as a potential reservoir for human infections. The pigeon farm was found to be an important reservoir for the fos $A 3$ gene and this should be further monitored.
\end{abstract}

Keywords: fosfomycin resistance; pigeon farm; E. coli; fos A3; transmission

\section{Introduction}

The prevalence of bacterial resistance to multiple antibiotics has resulted in fewer treatment options for clinicians. Fosfomycin has emerged as a candidate antibiotic to treat infections caused by carbapenem-, tigecycline-, and polymyxin-resistant bacteria [1]. Resistance to fosfomycin is mediated by FosA enzymes in Gram-negative bacteria and there are more than 10 fos types, of which fos $A$ and fos $C 2$ are the primary ones found in the Enterobacteriaceae [2]. The fos $A 3$ gene has also been co-localized via IS26 with bla $a_{\mathrm{CTX}-\mathrm{M}}$ on epidemic plasmids frequently detected in E. coli that spread among humans, pets, and food animals [3,4]. However, fosfomycin is not approved for use in animals in China, although the fos $A 3$ gene has been detected in food animals as well as humans [5].

Pigeon breeding is popular and common in China, especially in Guangdong province, and domestic pigeons are found in all urban areas, with a population size estimated at several hundred million [6]. Moreover, the number of pigeons consumed in China has grown to three times as many as one decade ago [7]. Importantly, clinically healthy homing pigeons have served as an unnoticed reservoir for zoonotic bacteria that are spread 
by faeces [8]. Furthermore, the close relationships between humans and birds have contributed to the spread of infectious agents, including antibiotic-resistant bacteria [9], and are an epidemiological link to wild birds [8]. The global and clonal multidrug-resistant carbapenem-resistant Enterobacteriaceae (CRE) carried by pigeons therefore may be established in ecological niches that include humans, livestock, wildlife, pets, and insects [10].

The goal of the present work was to reveal the distribution and prevalence of fosfomycin -resistant $E$. coli isolates in domestic pigeons. Our results indicated that pigeons carrying fos A3-positive Escherichia coli were prevalent in pigeon farms and that E. coli of human origin are a potential reservoir for the fos $A 3$ gene.

\section{Materials and Methods}

\subsection{Bacterial Isolation, Fosfomycin-Resistant Gene Identification, and Antimicrobial} Susceptibility Testing

A total of 79 samples including pigeon faeces $(n=34)$, pigeon ceca $(n=12)$, pigeon pericardial fluid $(n=6)$, sewage $(n=18)$, dust $(n=3)$, and flies $(n=6)$ were collected from a pigeon farm in South China, Foshan City, Guangdong province, in 2016. Briefly, flies, sewage, and dust were collected as reported previously [11]. Samples were incubated in Luria-Bertani (LB) liquid medium overnight and the fosfomycin-resistant isolates were selected on MacConkey agar containing $256 \mathrm{mg} / \mathrm{L}$ fosfomycin. All colonies were selected and identified using Gram staining and sequence analysis of the 16S rDNA gene using previously described primers [12]. Species identification of Gram-negative bacteria was further conducted by MALDI-TOF MS [13]. Fosfomycin resistance genes, including fos $A$, fos $A 3$, fos $C 2$, and bla $a_{\mathrm{CTX}-\mathrm{M}}$, were screened using PCR and sequencing [14]. Multilocus sequence typing (MLST) for E. coli was based on seven housekeeping genes, as reported previously [15]. PCR amplification and sequencing were performed following the protocols suggested on the website [16].

\subsection{Antimicrobial Susceptibility Testing}

The minimum inhibitory concentrations (MICs) of a range of antibiotics against the fosfomycin-resistant isolates were determined using the agar dilution method according to the Clinical and Laboratory Standards Institute (CLSI) document M100-S28 [17], and the breakpoints of colistin and tigecycline for Enterobacteriaceae were interpreted according to EUCAST criteria (Version 7.0) [18]. The E. coli isolate ATCC 25922 was used for quality control.

\subsection{Molecular Analysis of Fosfomycin-Resistant E. coli}

The fosfomycin-resistant $E$. coli were investigated by $\mathrm{XbaI}$ pulsed field electrophoresis (PFGE) and the locations of the fosfomycin resistance genes in the original isolates were determined by S1-PFGE mapping and Southern blot analysis, as described previously [19]. The PFGE patterns were analysed using a dendrogram of per cent similarity calculated with Dice coefficients using a cut-off of $80 \%$ [20]. The transferability of fosfomycin resistance genes was determined by the filter mating method using streptomycin-resistant $E$. coli C600 as a recipient strain, in accordance with a previous study [19].

\subsection{Whole-Genome Sequencing and Phylogenetic Analysis of fosA3-Positive Isolates}

Total DNA was extracted using a DNA Extraction Kit (TianGen, Beijing, China) and subjected to whole-genome sequencing (WGS). The library was constructed using the NEXT Ultra DNA Library Prep Kit (New England Biolabs, Ipswich, UK) and an Illumina HiSeq 2500 system (Novogene, Guangzhou, China), which produced 250 bp paired-end reads. A draft assembly of the sequences was generated using CLC Genomics Workbench 5 (CLC Bio, Aarhus, Denmark). All contigs were searched for fos $A 3$ genes using standalone BLAST analysis [21].

To explore the molecular relationships among the isolates in the current study and other resources, WGS information for 130 E. coli isolates was downloaded from GenBank as 
of 7 November 2020. (Table S1). The 130 representative isolates were selected according to the following standard. Briefly, the WGS of a total of 774,435 isolates were downloaded from GenBank and the fosA3-positive E. coli were screened using stand-alone BLAST analysis, resulting in a total of 915 fosA3-positive E. coli. Phylogenetic analysis was performed using the isolates from the current study and the public repository. Since the phylogenetic software could only process 500 isolates at a time, these 915 fosA3-positive E. coli strains were randomly divided into two groups and each group was employed to construct a phylogenetic tree with the 14 isolates in the current study, respectively. Then, the 130 representative strains were selected according to their distribution in the phylogenetic tree, sources, and sampling locations (Figure S2). MLST results were analysed using MLST Version 2 [22].

The downloaded genomic sequences, along with time and geospatial information, were applied to construct phylogenetic trees with the E. coli isolates using Parsnp in Harvest package 5 [23]. SNP assignments were determined using Snippy v4.4.5 [24] and Snippy-core was used to determine the core SNPs. For the phylogenetic tree, a reference genome was randomly selected using the "-r!" switch. The E. coli population structure was estimated with the hierBAPS module from the software BAPS v6.0 [25], which fits lineage to genome data using nested clustering. Annotations for each isolate and tree embellishment were visualized using iTOL [26].

\subsection{Data Availability}

Genome assemblies of 14 strains co-harbouring fos $A 3$ were deposited in GenBank under BioProject accession number PRJNA728561.

\subsection{Ethical Statement}

This research was conducted in accordance with the regulations of the ethical guidelines of South China Agricultural University. Written informed consent was obtained from the owners for the participation of pigeons.

\section{Results}

\subsection{Identification of fos Genes in Samples}

We identified a total of $69 \mathrm{E}$. coli isolates in our 79 samples collected on the pigeon farm that were resistant to fosfomycin (MIC $\geq 256 \mathrm{mg} \mathrm{L}^{-1}$ ), and these included isolates from faeces $(n=34)$, ceca $(n=12)$, liquor pericardii $(n=5)$, sewage $(n=14)$, dust $(n=1)$ and flies $(n=3)$. These isolates were further identified by $16 \mathrm{~S}$ rDNA gene sequencing combined with MALDI-TOF MS as E. coli $(n=52)$, Proteus mirabilis $(n=15)$ and Citrobacter freundii $(n=2)$. PCR results indicated that the prevalence of fos $A 3$ in the E. coli was $94.2 \%(49 / 52)$ and fos $A$ and fos $C$ variants were not detected. MLST analysis of these fos $A 3$-positive E. coli indicated nine types and ST6856 was the most prevalent sequence type (ST) $(24.5 \%, 12 / 49)$, followed by ST8804 (18.4\%, 9/49), ST457 (16.3\%, 8/49), ST746 (12.2\%, 6/49), ST533 (10.2\%, 5/49), ST165 (8.2\%, 4/49), ST2614 (4.1\%, 2/49), ST362 (4.1\%, 2/49) and ST8805 (2.0\%, 1/49).

The 49 fos A3-positive E. coli isolates were resistant to ampicillin, cefotaxime, fosfomycin, tetracycline and ciprofloxacin $(100 \%, 49 / 49)$, colistin $(95.9 \%, 47 / 49)$, trimethoprim/sulfamethoxazole $(73.47 \%, 36 / 49)$, florfenicol $(34.69 \%, 17 / 49)$, gentamicin $(18.37 \%$, 9/49) and amikacin $(2.04 \%, 1 / 49)$. All E. coli isolates were susceptible to chloramphenicol, meropenem and tigecycline and the fosfomycin-resistant $E$. coli remained highly susceptible to meropenem and tigecycline.

Conjugation experiments were performed on 49 fos $A 3$-positive $E$. coli isolates and generated 31 transconjugants $(63.27 \%, 31 / 49)$. For the 49 fos A3-positive E. coli isolates, PFGE was successfully performed and generated 13 pulsotypes designated as pulsotypes A-M. Pulsotype C, which was derived from faeces and sewage, as well as pulsotype I, from faeces, sewage and ceca, were the most prevalent clonal isolates and accounted for $18.4 \%$ of the total. Identical PFGE patterns were found between pericardial effusion and flies for pulsotype B as well as from faeces and sewage in pulsotypes E, G, I and K. Pulsotypes K-M 
were identified as ST457 and pulsotypes H-J as ST6856, whereas each PFGE pattern in the remaining pulsotypes was identified as one type of ST (Figure 1). Among these 49 isolates, S1-nuclease PFGE mapping and Southern blot analysis were performed successfully in $31(63.2 \%, 31 / 49)$ of them and revealed that the fos $A 3$ gene was present on differently sized plasmids, including $150 \mathrm{~kb}(n=10), 80 \mathrm{~kb}(n=5), 210 \mathrm{~kb}(n=7), 240 \mathrm{~kb}(n=1), 310 \mathrm{~kb}$ $(n=5), 340 \mathrm{~kb}(n=1)$ and $370 \mathrm{~kb}(n=2)$ (Figure S1).

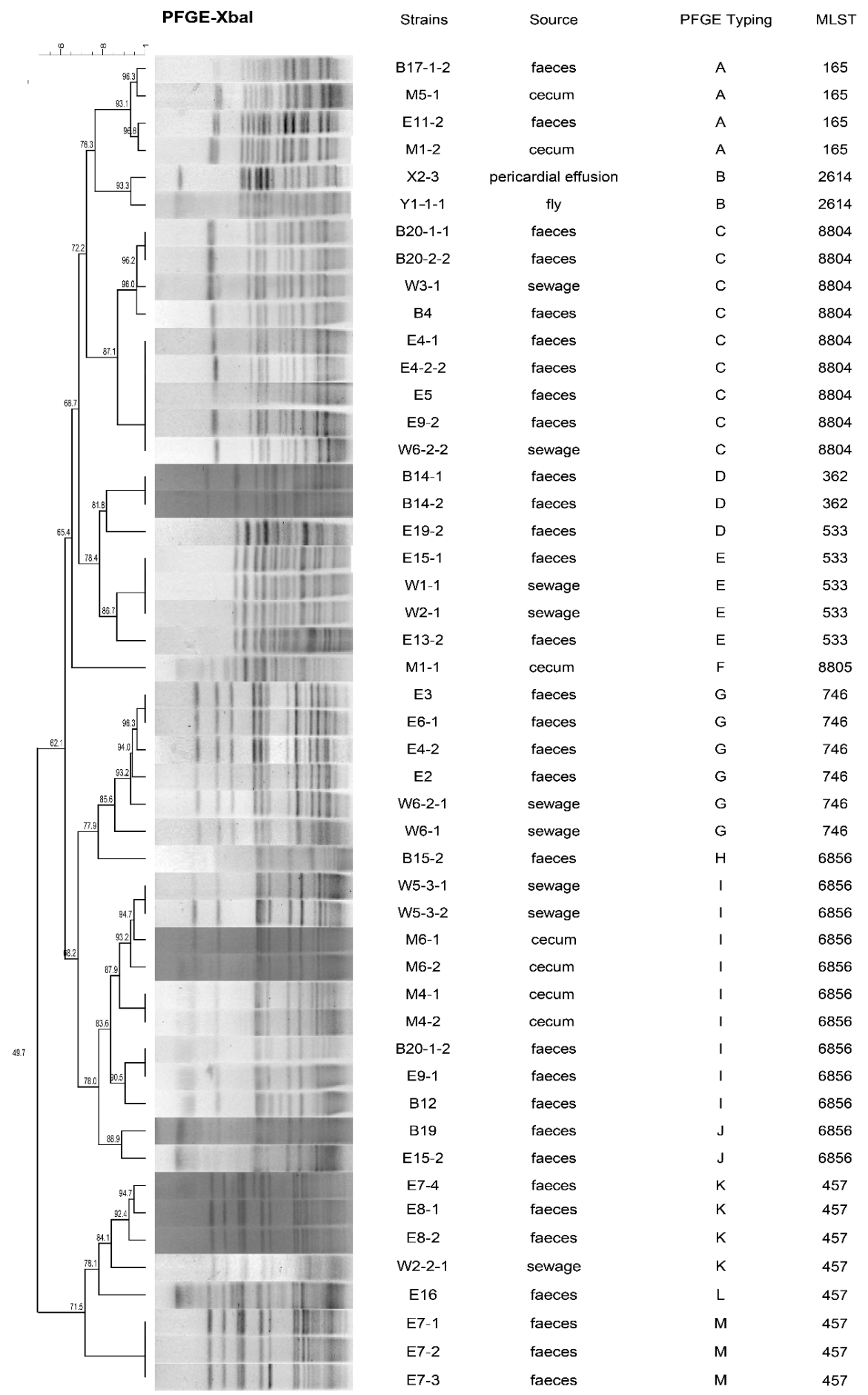

Figure 1. PFGE typing of 49 strains of fosA3-positive E. coli. Strains, sources and PFGE types are indicated, including 13 pulsotypes (A-M) that had similarities $>80 \%$. 


\subsection{Whole-Genome Sequencing and Phylogenetic Analysis of fosA3-Positive E. coli Isolates}

We performed WGS on two isolates each for pulsotypes $C$ and I, which possessed the largest numbers of isolates, and for single representatives from each of the remaining pulsotypes; only one failed to be sequenced. A total of 130 representative strains with time and geospatial information were then combined with these 14 isolates and a phylogenetic tree was constructed [27]. These 144 isolates were primarily distributed in China $(n=126)$, followed by South America $(n=7)$ and other counties $(n=11)$, and were derived from animals $(n=57)$, humans $(n=71)$ and environmental sources $(n=16)$ (Figure 2). These isolates were further clustered into eight groups sharing a total 163,219 SNPs. MLST analysis revealed that $f o s A 3$ was distributed across a diverse range of STs $(n=71)$. Cluster 2 contained only a single isolate (ST245) and cluster 6 possessed five isolates (all ST457). However, all other clusters contained $>2$ STs, such as cluster 1 which was dominated by ST167 (14/144), while ST167 (6/144) and ST156 (14/144) were prevalent in cluster 8. Additionally, the strain E7-1 from pigeon faeces shared only 2 SNPs with the strain W1-1 from a sewage isolate in cluster 8 and the strain GZEC065 from human blood shared only 18 SNPs with the strain WFA04 from pig lungs. A strain from a chicken cecum shared only one SNP with strain 648 from a dog anal swab sample (Figure 2).

We identified the presence of 40 antibiotic resistance genes (ARGs) which mediated resistance to 11 types of antibiotics that co-existed with fos $A 3$ in these 144 E. coli isolates. These included resistance genes to fosfomycin, $\beta$-lactams, colistin, tetracycline, aminoglycosides, chloramphenicol, quinolones, macrolides, sulphonamides, trimethoprim, and rifampicin (antibiotic resistance gene class is represented with the line colours at the bottom of Figure 2). The $\beta$-lactamase genes displayed the greatest diversity and bla $a_{\mathrm{CTX}-\mathrm{M}}$ $(91 \%, 131 / 144)$ and $m d f A(98.6 \%, 142 / 144)$ were most prevalent. The genes $b l a_{\mathrm{NDM}}, \mathrm{mcr}$ and tet $(\mathrm{X} 4)$, which mediate carbapenem, colistin, and tigecycline resistance, were present among these fosA3-carrying E. coli at 38.9, 77.8 and $0.69 \%$, respectively.

\subsection{Genetic Environments}

A comparative genomic analysis indicated that fos $A 3$ was present in three genomic contexts including types I, III and V, with I being the most prevalent, which originated from faeces, ceca and sewage. The entries from the fos $A 3$-positive $E$. coli from the public repository indicated that three genomic environments flanking fos $A 3$ in $E$. coli of human origin resembled those found in current study (types II, IV and VI). Type III of pigeon origin and type IV of human origin shared the greatest similarities in the backbones and differed only by one IS26 element, which was complete in type IV but truncated in type III. In the remaining four genomic contexts, IS26 was present both upstream and downstream of the fos $A 3$ gene. In addition, the extended spectrum $\beta$-lactamase (ESBL) gene $b l a_{\mathrm{CTX}-\mathrm{M}}$ was frequently located upstream of the fos $A 3$ gene in these six genomic contexts (Figure 3 ). In addition, combining the NCBI database information (998 fos A3-positive E. coli isolates downloaded on 7 November 2020) with the WGS (14 fosA3-positive E. coli isolates) analysis of this study, we found that bla $a_{\text {CTX-M }}$ was present in $92.59 \%(937 / 1012)$ of the isolates. The prevalence of $b l a_{\mathrm{CTX}-\mathrm{M}-14}, b l a_{\mathrm{CTX}-\mathrm{M}-15}, b l a_{\mathrm{CTX}-\mathrm{M}-55}$ and $b l a_{\mathrm{CTX}-\mathrm{M}-65}$ was $27.17,4.94,36.67$ and $17.292 \%$, respectively. 


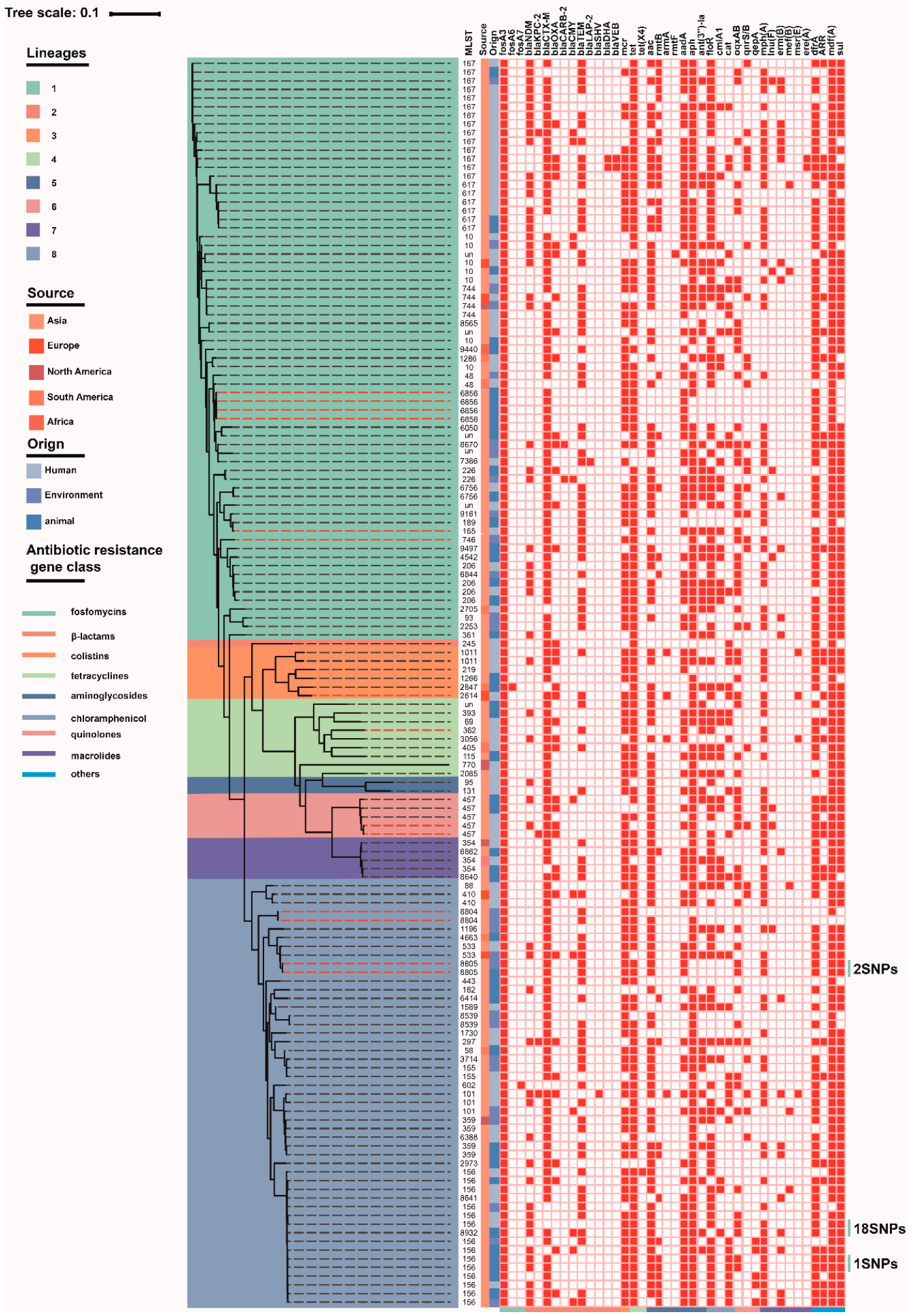

Figure 2. Phylogenetic tree along with ARGs present in the E. coli isolates. The phylogenetic tree is composed of 144 fos A3positive E. coli divided into eight clusters as indicted. The strains are marked with an orange dashed line in this study. 


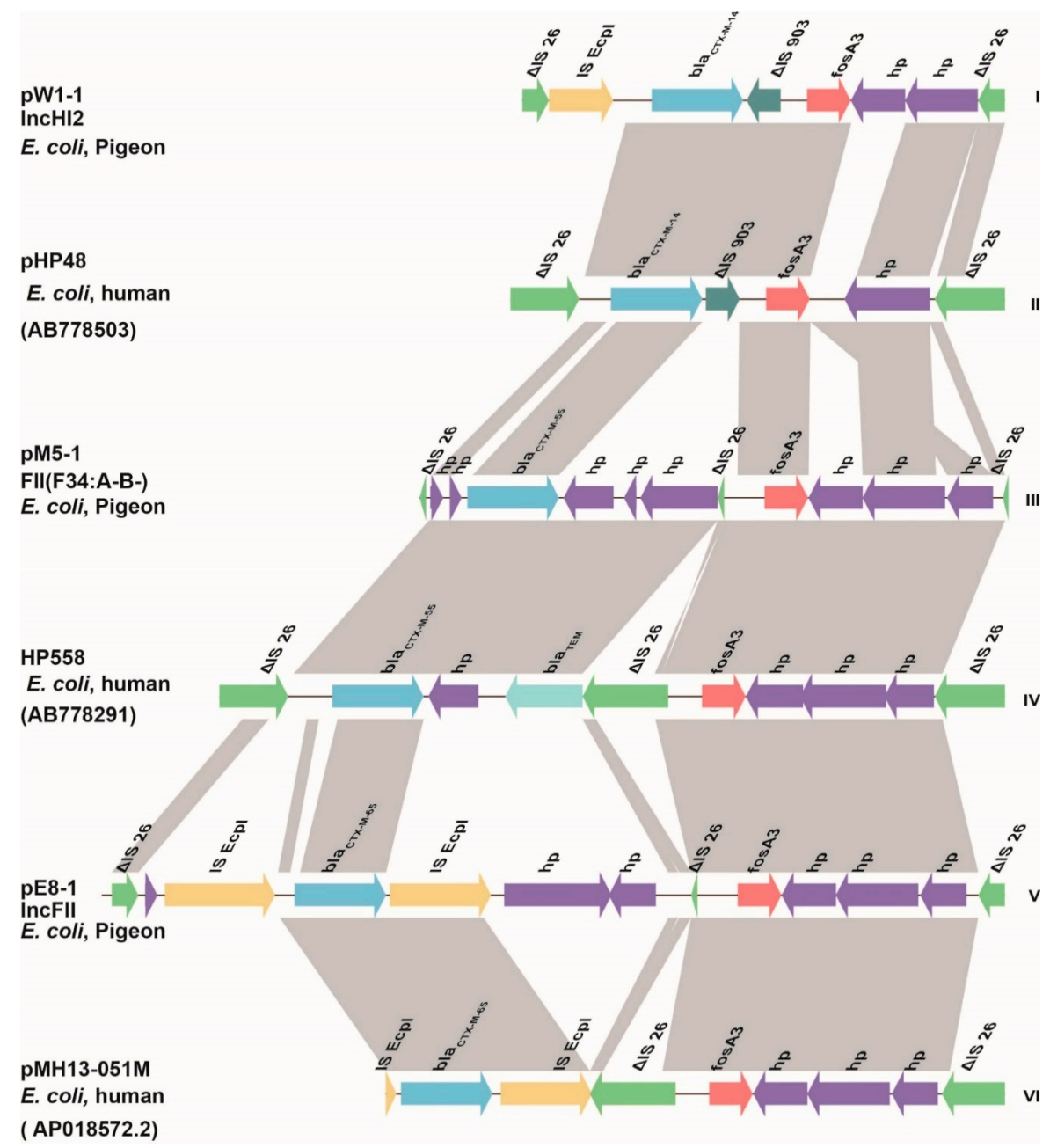

Figure 3. Genetic environment of fos $A 3$ in 14 E. coli isolates. Arrows indicate the directions of transcription of the genes, and different genes are shown in different colours. Regions of $>99.0 \%$ nucleotide sequence identity are shaded in grey. The delta $(\Delta)$ symbol indicates a truncated gene.

\section{Discussion}

E. coli resistant to fosfomycin have become a serious global health problem $[28,29]$. The high level $(71 \%, 49 / 69)$ of fos $A 3$-positive E. coli detected from pigeons in the current study was significantly higher than that found for pets $(10.34 \%, 3 / 29)$ and food animals $(1.1 \%, 10 / 892)$ in previous studies for Guangzhou, China [30-32]. PFGE patterns combined with the genomic contexts of regions flanking fos $A 3$ indicated that fos $A 3$ could spread by horizontal transfer as well as via clonal dissemination between $E$. coli on the pigeon farm, and this most likely led to its high prevalence.

Our isolates also frequently contained IS 26 both upstream and downstream of fos $A 3$ and this has been previously reported [33]. This is further evidence that IS26 plays a role in the dissemination of $f o s A 3$ in E. coli. IS26 also facilitated ARG diversity in our isolates, including aph $A 1, \operatorname{tet}(\mathrm{C}), \operatorname{tet}(\mathrm{D}), \operatorname{cat} A 2$ and $\operatorname{cfr}[34]$. The $f o s A 3$ gene was generally found co-localized with $b l a_{\mathrm{CTX}-\mathrm{M}}[34]$ and this most likely was the result of the combined administration of fosfomycin and $\beta$-lactam antibiotics that promoted their co-transfer between Gram-negative bacteria.

The use of fosfomycin in food-producing animals has not been approved in China. Nevertheless, we found a high prevalence of fos $A 3$ in food-producing animals and in E. coli, P. mirabilis, E. fergusonii and C. freundii isolates from pets, and pet owners [4]. The isolates sharing $<35$ SNPs were classified as clonally spread according to criteria defined in a previous study $[35,36]$. The phylogeny for a strain from human blood (accession: 
SAMN13875146) that shared only 18 SNPs with strain WFA04 from a pig suggested that the fos $A 3$-carrying $E$. coli were likely spread between humans and food animals via clonal propagation. In addition, we found that the genomic contexts that flanked fos $A 3$ in the current study closely resembled those from human isolates, suggesting that the pigeon farm served as a potential reservoir for human infections.

\section{Conclusions}

In current study, we detected a high prevalence of fos $A 3$ in pigeon farm samples and found that $f o s A 3$ could spread by both horizontal and clonal transfer. The high similarity of the fos $A 3$ genomic environment between $E$. coli isolates from humans and pigeons indicated that the pigeon farm served as a reservoir for these strains. The presence of fos $A 3$ on the pigeon farm poses a threat to public health and should be regularly monitored.

Supplementary Materials: The following are available online at https:/ / www.mdpi.com/article/10 .3390 / antibiotics10070777/s1, Figure S1: S1-nuclease PFGE mapping and Southern blot analysis of fosA3-carrying plasmids on different sizes. Figure S2: Phylogenetic analysis of the fosA3 positive E. coli from public repository and current study Table S1: Sample collection, strain identification and MIC results.

Author Contributions: Data curation, L.H. and Y.X.; Formal analysis, L.H. and Y.X.; Funding acquisition, X.-P.L., Y.-H.L. and J.S.; Investigation, L.H., X.-Q.L., X.-W.L. and R.-Y.S.; Methodology, R.-M.Z.; Project administration, Y.-H.L. and J.S.; Resources, L.H.; Software, L.H., M.-N.L. and R.-Y.S.; Writing—original draft, L.H.; Writing—review \& editing, R.-M.Z. All authors have read and agreed to the published version of the manuscript.

Funding: This work was supported in part by the Program for Innovative Research Team of the University of the Ministry of Education of China (grant IRT_17R39), the Local Innovative and Research Teams Project of Guangdong Pearl River Talents Program (grant 2019BT02N054) and the 111 Project (grant D20008).

Data Availability Statement: The data for this manuscript is available from correspondence author.

Conflicts of Interest: The authors declare no conflict of interest.

\section{References}

1. Lletí, J.R.R. Fosfomycin in infections caused by multidrugresistant Gram-negative pathogens. Soc. Esp. Quimioter. 2019, 32, 45-54.

2. Yang, T.Y.; Lu, P.L.; Tseng, S.P. Update on fosfomycin-modified genes in Enterobacteriaceae. J. Microbiol. Immunol. Infect. 2017, 52, 9-21. [CrossRef]

3. He, D.; Liu, L.; Guo, B.; Wu, S.; Chen, X.; Wang, J.; Zeng, Z.; Liu, J. Chromosomal location of the fosA3 and bla Proteus mirabilis and clonal spread of Escherichia coli ST117 carrying fosA3-positive IncHI2/ST3 or F2: A-: B-plasmids in a chicken farm. Int. J. Antimicrob. Agents 2017, 49, 443-448. [CrossRef]

4. Yao, H.; Wu, D.; Lei, L.; Shen, Z.; Wang, Y.; Liao, K. The detection of fosfomycin resistance genes in Enterobacteriaceae from pets and their owners. Vet. Microbiol. 2016, 193, 67-71. [CrossRef] [PubMed]

5. Zhang, L.; Gu, X.; Zhang, J.; Yang, L.; Lu, Y.; Fang, L.; Jiang, H. Characterization of a fosA3 carrying IncC-IncN plasmid from a multidrug-resistant ST17 Salmonella Indiana isolate. Front. Microbiol. 2020, 11, 1582. [CrossRef]

6. Haag-Wackernagel, D. Die taube-eine erfolgsgeschichte. vom liebling der götter zur eroberung der städte. Biol. Unserer Zeit 2011, 41, 44-52. [CrossRef]

7. Ye, M.; Xu, M.; Chen, C.; He, Y.; Ding, M.; Ding, X.; Wei, W.; Yang, S.; Zhou, B. Expression analyses of candidate genes related to meat quality traits in squabs from two breeds of meat-type pigeon. J. Anim. Physiol. Anim. Nutr. 2017, 102, 727-735. [CrossRef]

8. Teske, L.; Ryll, M.; Rubbenstroth, D.; Hänel, I.; Hartmann, M.; Kreienbrock, L.; Rautenschlein, S. Epidemiological investigations on the possible risk of distribution of zoonotic bacteria through apparently healthy homing pigeons. Avian Pathol. 2013, 42, 397-407. [CrossRef] [PubMed]

9. Mukerji, S.; Gunasekera, S.; Dunlop, J.N.; Stegger, M.; Jordan, D.; Laird, T.; Abraham, R.J.; Barton, M.; O’Dea, M.; Abraham, $\mathrm{S}$. Implications of foraging and interspecies interactions of birds for carriage of escherichia coli strains resistant to critically important antimicrobials. Appl. Environ. Microbiol. 2020, 86, e01610-e01620. [CrossRef]

10. Zhang, R.-M.; Liu, X.; Wang, S.-L.; Fang, L.-X.; Sun, J.; Liu, Y.-H.; Liao, X.-P. Distribution patterns of antibiotic resistance genes and their bacterial hosts in pig farm wastewater treatment systems and soil fertilized with pig manure. Sci. Total Environ. 2020, 758, 143654. [CrossRef] [PubMed] 
11. Wang, Y.; Zhang, R.; Li, J.; Wu, Z.; Yin, W.; Schwarz, S.; Tyrrell, J.M.; Zheng, Y.; Wang, S.; Shen, Z.; et al. Comprehensive resistome analysis reveals the prevalence of NDM and MCR-1 in Chinese poultry production. Nat. Microbiol. 2017, 2, 16260. [CrossRef]

12. Walsh, F.; Duffy, B. The culturable soil antibiotic resistome: A community of multi-drug resistant bacteria. PLoS ONE 2013, 8, e65567. [CrossRef] [PubMed]

13. Seng, P.; Drancourt, M.; Gouriet, F.; La Scola, B.; Fournier, P.; Rolain, J.M.; Raoult, D. Ongoing revolution in bacteriology: Routine identification of bacteria by matrix-assisted laser desorption ionization time-of-flight mass spectrometry. Clin. Infect. Dis. 2009, 49, 543-551. [CrossRef] [PubMed]

14. Sato, N.; Kawamura, K.; Nakane, K.; Wachino, J.; Arakawa, Y. First detection of fosfomycin resistance gene fosA3 in CTXM-producing Escherichia coli isolates from healthy individuals in Japan. Microb. Drug Resist. 2013, 19, 477-482. [CrossRef] [PubMed]

15. Wirth, T.; Falush, D.; Lan, R.; Colles, F.; Mensa, P.; Wieler, L.H.; Karch, H.; Reeves, P.R.; Maiden, M.C.J.; Ochman, H.; et al. Sex and virulence in Escherichia coli: An evolutionary perspective. Mol. Microbiol. 2006, 60, 1136-1151. [CrossRef]

16. Escherichia coli Sequence Typing. Available online: https://bigsdb.pasteur.fr/ecoli/ecoli.htm (accessed on 27 August 2016).

17. CLSI. Performance Standards for Antimicrobial Susceptibility Testing-Twenty-Eighth Edition; CLSI: Annapolis Junction, MD, USA, 2018; p. M100.

18. EUCAST. Breakpoint Tables for Interpretation of MICs and Zone Diameters. Version 7.0; EUCAST: Växjö, Sweden, 2017.

19. Zhang, R.; Wan, Y.; Liu, Z.; Li, J.; Yin, W.; Lei, L.; Wu, C.; Shen, J. Characterization of NDM-1-producing carbapenemase in Acinetobacter spp. and E. coli isolates from diseased pigs. Front. Agric. Sci. Eng. 2015, 2, 223. [CrossRef]

20. Wang, M.; Zhang, R.; Wang, L.; Sun, R.; Bai, S.; Han, L.; Fang, L.; Sun, J.; Liu, Y.; Liao, X. Molecular epidemiology of carbapenemase-producing Escherichia coli from duck farms in south-east coastal China. J. Antimicrob. Chemother. 2020, 76, 322-329. [CrossRef]

21. Madden, T. The BLAST Sequence Analysis Tool. 2013. Available online: https://blast.ncbi.nlm.nih.gov/Blast.cgi (accessed on 15 March 2013).

22. Inouye, M.; Harriet Dashnow, L.A.R.; Schultz, M.B.; Pope, B.J.; Tpmita, T.; Zobel, J.; Holt, K.E. SRST2: Rapid genomic surveillance for public health and hospital microbiology labs. Genome Med. 2014, 6, 90. [CrossRef]

23. Treangen, T.J.; Ondov, B.D.; Koren, S.; Phillippy, A.M. The Harvest suite for rapid core-genome alignment and visualization of thousands of intraspecific microbial genomes. Genome Biol. 2014, 15, 524. [CrossRef]

24. Tseemann. Snippy (Version 4.4.5). Available online: https://github.com/tseemann/snippy (accessed on 4 January 2020).

25. Cheng, L.; Connor, T.R.; Sirén, J.; Aanensen, D.M.; Corander, J. Hierarchical and spatially explicit clustering of DNA sequences with BAPS software. Mol. Biol. Evol. 2013, 30, 1224-12248. [CrossRef]

26. Letunic, I.; Bork, P. Interactive tree of life (iTOL) v4: Recent updates and new developments. Nucleic Acids Res. 2019, 47, W256-W259. [CrossRef] [PubMed]

27. Sanaa, A.A.; Lo, C.-C.; Li, P.-E.; Davenport, K.D.; Chain, P.S.G. Chain. From raw reads to trees: Whole genome SNP phylogenetics across the tree of life. bioRxiv 2015. [CrossRef]

28. Lei, C.-H.; Chen, Y.-A.; Kang, Z.H.; Kong, L.I.; Wang, H.-O. Characterization of a Novel SXT/R391 integrative and conjugative element carrying cfr, $b l a_{\mathrm{CTX}-\mathrm{M}-65}$, fos A3, and aac $\left(6^{\prime}\right)-\mathrm{Ib}-\mathrm{cr}$ in Proteus mirabilis. Antimicrob. Agents Chemother. 2018, 62, e00849-18. [CrossRef]

29. Cyoia, C.P.S.; Koga, V.L.; Nishio, E.K.; Houle, S.; Dozois, C.M.; de Brito, K.C.T.; de Brito, B.G.; Nakazato, G.; Kobayashi, R.K.T. Distribution of ExPEC virulence factors, bla CTX-M $_{1}$ fos $A 3$, and $m c r-1$ in Escherichia coli isolated from commercialized chicken carcasses. Front. Microbiol. 2018, 9, 3254. [CrossRef]

30. Hou, J.; Huang, X.; Deng, Y.; He, L.; Yang, T.; Zeng, Z.; Chen, Z.; Liu, J. Dissemination of the fosfomycin resistance gene fosA3 with CTX-M $\beta$-lactamase genes and $r m t B$ carried on IncFII Plasmids among Escherichia coli isolates from pets in China. Antimicrob. Agents Chemother. 2011, 56, 2135-2138. [CrossRef]

31. Hou, J. Detection of the plasmid-encoded fosfomycin resistance gene fosA3 in Escherichia coli of food-animal origin. J. Antimicrob. Chemother. 2012, 68, 766-770. [CrossRef]

32. Ho, P.L.; Chan, J.; Lo, W.U.; Law, P.Y.; Li, Z.; Lai, E.L.; Chow, K.H. Dissemination of plasmid-mediated fosfomycin resistance fosA3 among multidrug-resistant Escherichia coli from livestock and other animals. J. Appl. Microbiol. 2013, 114, 695-702. [CrossRef]

33. Partridge, S.R.; Kwong, S.M.; Firth, N.; Jensen, S.O. Mobile genetic elements associated with antimicrobial resistance. Clin. Microbiol. Rev. 2018, 31, e00088-17. [CrossRef]

34. Cheng, K.; Fang, L.; Ge, Q.; Wang, D.; He, B.; Lu, J.; Zhong, Z.; Wang, X.; Yu, Y.; Lian, X.; et al. Emergence of fosA3 and bla CTX-M-14 in multidrug-resistant citrobacter freundii isolates from flowers and the retail environment in China. Front. Microbiol. 2021, 12, 586504. [CrossRef]

35. David, S.; Reuter, S.; Harris, S.R.; Glasner, C.; Feltwell, T.; Argimon, S.; Abudahab, K.; Goater, R.; Giani, T.; Errico, G.; et al. Epidemic of carbapenem-resistant Klebsiella pneumoniae in Europe is driven by nosocomial spread. Nat. Microbiol. 2019, 4, 1919-1929. [CrossRef]

36. Chew, K.L.; Octavia, S.; Ng, O.T.; Marimuthu, K.; Venkatachalam, I.; Cheng, B.; Lin, R.T.P.; Teo, J.W.P. Challenge of drug resistance in Pseudomonas aeruginosa: Clonal spread of NDM-1-positive ST-308 within a tertiary hospital. J. Antimicrob. Chemother. 2019, 74, 2220-2224. [CrossRef] 